\title{
Intakes of omega-3 polyunsaturated fatty acids in an Irish pre-school population
}

\author{
Y. M. Lenighan, B. A. McNulty, N. F. C. Devlin, M. J. Gibney and A. P. Nugent \\ UCD Institute of Food and Health, University College Dublin, Belfield, Dublin 4, Ireland
}

Omega-3 polyunsaturated fatty acids have been positively associated with cognitive and visual development in infants and young children $^{(1)}$. There is no global consensus regarding recommended intakes in pre-schoolers, although EFSA advise a mean daily intake of $0.5 \%$ of total energy (TE) ALA and $100 \mathrm{mg} / \mathrm{d}$ DHA for children aged 6-24 months ${ }^{(2)}$. For adults, a combined intake of $\geqslant 250 \mathrm{mg} / \mathrm{d}$ EPA \& DHA is recommended ${ }^{(2)}$. Currently, there is no data on intakes of omega-3 PUFA for Irish pre-school children, therefore this analysis aimed to characterise intakes of ALA, EPA and DHA using data from the National Pre-school Nutrition Survey (NPNS) ${ }^{(3)}$. The NPNS examined habitual food and beverage intakes of 500 Irish children aged 1-4 years during 2010-2011 ${ }^{(3)}$. In this analysis, the NPNS database was updated for fatty acid content $(\mathrm{g} / 100 \mathrm{~g})$ using manufacturer's information and published food composition databases. Mean daily intakes and compliance with EFSA recommendations were assessed and the main food sources contributing to omega-3 PUFA intakes identified.

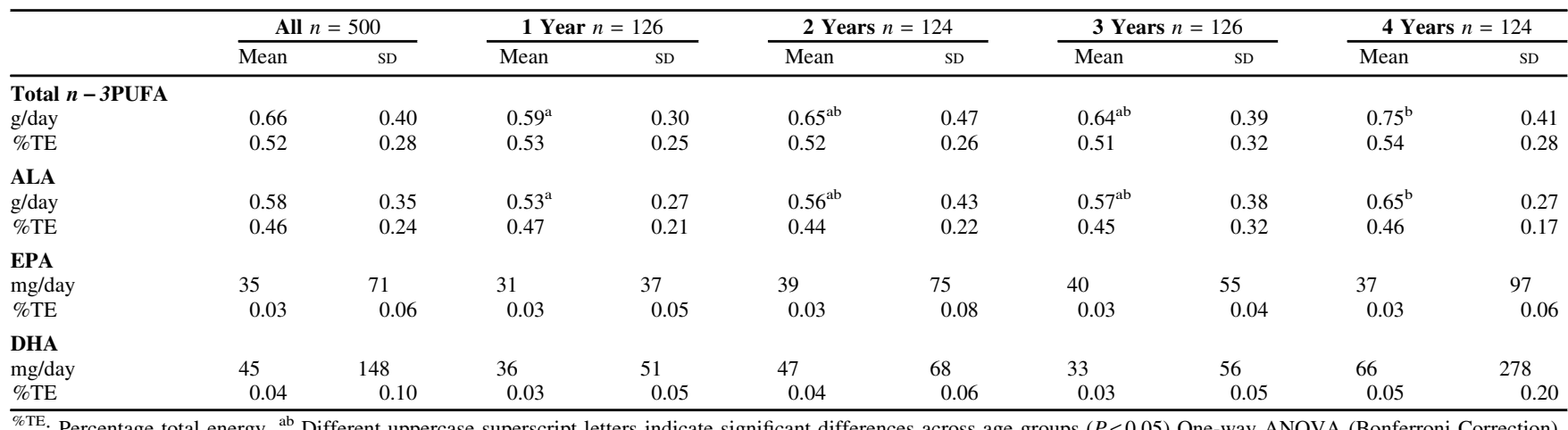

Intakes of total $n-3$ PUFA and ALA increased significantly with age (g/d) but not as a \%TE. Intakes of EPA and DHA were typically low (EPA, 31-40 mg; DHA, 36-66 mg). Similar patterns were observed when the dataset was split by gender. There was high compliance with the EFSA ALA recommendation $(\leqslant 82.2 \%)$ but poor adherence with the age-specific recommendation for DHA in 1 year olds $(\leqslant 27.7 \%)$ and the comparable adult recommendation $(\leqslant 23.2 \%)^{(2)}$. The main source of ALA $(15.1 \%)$ was the food group 'other' which included nuts and seeds while 'fish, fish products and fish dishes' was the primary source of EPA (33.7\%) and DHA (31.1\%). Nevertheless, only $44-59 \%$ of participants consumed fish during the survey period ${ }^{(3)}$, while $20 \%$ consumed supplements of which only $12 \%$ contained these fatty acids ${ }^{(3)}$. These results indicate that intakes of ALA were adequate, while intakes of EPA and DHA were inadequate. Effective strategies are needed to improve intakes of EPA and DHA in this young population which could include education, fortification and/or enrichment.

The project was funded by DAFF under the FHRI initiative 2007-2012.

1. Connor WE (2000) Am J Clin Nutr 71, 171S-175S.

2. EFSA Panel on Dietetic Products Nutrition and Allergies (2010) EFSA Journal 8(3), 1461.

3. IUNA (2012) National Pre-School Nutrition Survey. Summary Report on: Food and Nutrient Intakes, Physical Measurements and Barriers to Healthy Eating. 